\title{
A COMMUTATIVITY THEOREM FOR UNBOUNDED OPERATORS IN HILBERT SPACE $\left({ }^{1}\right)$
}

\author{
BY \\ A. E. NUSSBAUM
}

1. Introduction. B. Fuglede [3] has shown that if a bounded (everywhere defined linear) operator $B$ permutes with a normal operator $A$, i.e. $B A \subset A B$, then the spectral projections of $A$ permute with $B$. That is, $B E(\sigma)=E(\sigma) B$ for all Borel sets $\sigma$, where $\{E(\sigma)\}$ is the canonical spectral measure of $A$.

The purpose of this note is to prove a commutativity theorem for unbounded operators in Hilbert space which is an extension of Fuglede's theorem. It states in essence that if $N$ is a normal operator and $A$ a densely defined linear operator which has a closure (i.e., $A^{*}$ is densely defined), $D(N) \subset D\left(A^{*}\right)$, and $N A x=A N x$ for an appropriate set of vectors $x$ (cf. Theorem 1), then the spectral measure of $N$ permutes with $\bar{A}$. That is, $E(\sigma) \bar{A} \subset \bar{A} E(\sigma)$, for all Borel sets $\sigma$, where $\{E(\sigma)\}$ is the canonical spectral measure of $N$.

Although the commutativity theorem proved in this paper seems rather special, it is general enough to enable us to derive several known and some new theorems as simple corollaries.

In connection with our theorem the following example due to B. Fuglede [6] is of interest: There exists a closed symmetric operator which is unitarily equivalent with a proper extension of itself. Thus there exists a unitary operator $U$ and a closed symmetric operator $S$ such that $U$ permutes with $S$ but the spectral measure of $U$ does not permute with $S$. We are indebted to Professor Fuglede for calling our attention to this reference.

If $T$ is a normal operator with canonical spectral measure $\{E(\sigma)\}$, we shall denote by $D_{0}(T)$ the union $\bigcup R(E(\sigma))$, as $\sigma$ ranges over all bounded Borel sets. $(R(E(\sigma))$ denotes the range of the spectral projection $E(\sigma)$.) Clearly $D_{0}(T)$ is contained in $D(T)$ and $T$ is the closure of its restriction $T_{0}$ to $D_{0}(T)$.

We shall have need for the following proposition.

Proposition 1. Let $A$ be a densely defined closed linear operator in a Hilbert space $\mathfrak{K}$ such that $D(A) \subset D\left(A^{*}\right)$ and $\|A x\|=\left\|A^{*} x\right\|$ for all $x$ in $D(A)$. Suppose $T$ is a normal operator such that $D_{0}(T) \subset D(A)$ and the spectral measure of $T$ permutes with $A$, then $A$ is normal. In particular $A$ is selfadjoint if it is symmetric.

Proof. It is sufficient to show that $D\left(A^{*}\right) \subset D(A)$ (cf. [4, p. 33]). Let $x \in D\left(A^{*}\right)$, $\sigma_{n}=\{\lambda|| \lambda \mid \leqq n\}$ and $x_{n}=E\left(\sigma_{n}\right) x$, where $\{E(\sigma)\}$ is the canonical spectral measure of $T$.

Received by the editors March 18, 1968 and, in revised form, August 27, 1968.

(1) This work was in part supported by National Science Foundation Grants N.S.F. GP3583 and GP-6907. 
Then $x_{n} \in D(A)$ for all $n, x_{n} \rightarrow x$ as $n \rightarrow \infty$, and

$$
\left\|A x_{n}-A x_{m}\right\|=\left\|A^{*} x_{n}-A^{*} x_{m}\right\|=\left\|E\left(\sigma_{n}\right) A^{*} x-E\left(\sigma_{m}\right) A^{*} x\right\| \rightarrow 0
$$

as $n, m \rightarrow \infty$. Hence, since $A$ is closed, $x \in D(A)$.

We recall that two normal operators are said to commute if their spectral projections permute. This definition is consistent with the usual one in case one of the operators is bounded by virtue of Fuglede's theorem.

For further reference we state the following proposition. The proof is easily supplied by the reader.

Proposition 2. Suppose $A$ and $B$ are normal operators in a Hilbert space $\mathfrak{S E}$ and $\mathfrak{S E}$ is the orthogonal direct sum of subspaces $\mathfrak{M}_{n}, n=1, \ldots$, and every $\mathfrak{M}_{n}$ reduces $A$ and $B$ to (normal) operators $A_{n}$ and $B_{n}$, respectively. If $A_{n}$ and $B_{n}$ permute for every $n$, then $A$ and $B$ permute.

\section{The main theorem.}

THEOREM 1. Let $N$ and $T$ be permuting normal operators in a Hilbert space $\mathfrak{S}$ and $A$ a densely defined linear operator in $\mathfrak{S}$. Suppose that

1. $D_{0}(T) \subset D(N) \subset D(A) \cap D\left(A^{*}\right)$ (or more generally $D_{0}(T) \subset D(N) \cap D(A)$ $\cap D\left(A^{*}\right)$ and $\left.D_{0}(N) \subset D\left(A^{*}\right)\right)$ and

2. $\left(N x, A^{*} y\right)=\left(A x, N^{*} y\right)$ for all $x$ and $y$ in $D_{0}(T)$.

Then the spectral measure of $N$ permutes with $\bar{A}$ (the closure of $A$ ).

Proof. We first show that the hypothesis of Theorem 1 implies that $D_{0}(T)$ is contained in $D(A N)$ and $D(N A)$, and that

$$
A N x=N A x \text { for all } x \text { in } D_{0}(T) .
$$

Indeed, let $\{E(\sigma)\}$ be the canonical spectral measure of $T$ and $x \in D_{0}(T)$. Then $x=E(\sigma) x$, for some bounded Borel set $\sigma$. Hence $N x=N E(\sigma) x=E(\sigma) N x \in D_{0}(T)$ $\subset D(A)$. Therefore $x \in D(A N)$ and

$$
(A N x, y)=\left(N x, A^{*} y\right)=\left(A x, N^{*} y\right) \text { for all } y \in D_{0}(T)
$$

by 2. Now, $N^{*}$ is the closure of its restriction to $D_{0}(T)$. For if $z \in D\left(N^{*}\right)$, let $z_{n}=E\left(\sigma_{n}\right) z$, where $\sigma_{n}=\{\lambda|| \lambda \mid \leqq n\}$. Then $z_{n} \in D_{0}(T)$ for all $n, z_{n} \rightarrow z$ and $N^{*} z_{n}$ $=N^{*} E\left(\sigma_{n}\right) z=E\left(\sigma_{n}\right) N^{*} z \rightarrow N^{*} z$ as $n \rightarrow \infty$.

From (2), and the fact that $N^{*}$ is the closure of its restriction to $D_{0}(T)$ follows

$$
(A N x, y)=\left(A x, N^{*} y\right) \text { for all } y \in D\left(N^{*}\right) .
$$

Hence $A x \in D(N)$ and $N A x=A N x$. This proves (1).

Since by $1 A^{*}$ is densely defined, $A$ has a closure $\bar{A}$ and therefore

$$
\bar{A} N x=N \bar{A} x \text { for all } x \in D_{0}(T)
$$

by (1). 
Let now $\{F(\tau)\}$ be the canonical spectral measure of $N$ and $\sigma$ be a bounded Borel set in $R^{2}$. Then

$$
\bar{A} N E(\sigma) x=N \bar{A} E(\sigma) x \text { for all } x \in \mathfrak{H}
$$

by (4). But $E(\sigma) N \subset N E(\sigma)$. Hence

by (5); i.e.,

$$
(\bar{A} E(\sigma)) N x=\bar{A} N E(\sigma) x=N(\bar{A} E(\sigma)) x \text { for all } x \in D(N)
$$

$$
(\bar{A} E(\sigma)) N \subset N(\bar{A} E(\sigma)) .
$$

Now, $\bar{A} E(\sigma)$ is a closed operator with domain $\mathfrak{S}$. Therefore by the closed graph theorem $\bar{A} E(\sigma)$ is bounded. Hence, it follows from (6) by Fuglede's theorem (cf. Introduction) that

$$
F(\Delta) \bar{A} E(\sigma)=\bar{A} E(\sigma) F(\Delta)
$$

for all Borel sets $\Delta$. Let now $\Delta$ be a bounded Borel set in (7) and take adjoints of both sides of (7). We obtain

$$
(F(\Delta) \bar{A} E(\sigma))^{*}=(\bar{A} E(\sigma) F(\Delta))^{*} \supset F(\Delta) E(\sigma) A^{*}=E(\sigma) F(\Delta) A^{*},
$$

since $T$ and $N$ permute. But $(F(\Delta) \bar{A} E(\sigma))^{*} \supset E(\sigma) A^{*} F(\Delta)$ and the domain of $E(\sigma) A^{*} F(\Delta)$ is $\mathfrak{S}$ by 1 . Hence,

$$
E(\sigma) A^{*} F(\Delta) \supset E(\sigma) F(\Delta) A^{*},
$$

where $\sigma$ and $\Delta$ are arbitrary bounded Borel sets. From (9) follows if we let $\sigma=\sigma_{n}$ $=\{\lambda|| \lambda \mid \leqq n\}$ and $n \rightarrow \infty$, that

$$
F(\Delta) A^{*} \subset A^{*} F(\Delta)
$$

for all bounded Borel sets $\Delta$. From (10) and the fact that $A^{*}$ is closed follows

$$
F(\tau) A^{*} \subset A^{*} F(\tau)
$$

for all Borel sets $\tau$. This is equivalent with $F(\tau) \bar{A} \subset \bar{A} F(\tau)$ for all Borel sets $\tau$.

CoRollary 1. Let $A$ be a densely defined linear operator in a Hilbert space such that $D(A) \subset D\left(A^{*}\right)$ and $\|A x\|=\left\|A^{*} x\right\|$ for all $x \in D(A)$. If $N$ is a normal operator such that $D_{0}(N) \subset D(A)$ and

$$
\left(N x, A^{*} y\right)=\left(A x, N^{*} y\right) \text { for all } x, y \in D_{0}(N),
$$

then $\bar{A}$ is normal and commutes with $N$. In particular $\bar{A}$ is selfadjoint and permutes with $N$ if $A$ is symmetric.

Proof. The proof is an immediate consequence of Theorem 1 and Proposition 1.

\section{Applications.}

THEOREM 2 [2]. If $N, N_{1}$ and $N_{2}$ are normal operators such that $N=N_{1} N_{2}=N_{2} N_{1}$, then $N_{1}$ and $N_{2}$ permute. 
Proof. Let $T=N^{2}$. Then $T$ is normal and permutes with $N$. Moreover $D(T)$ $\subset D(N) \subset D\left(N_{1}\right)=D\left(N_{1}^{*}\right)$ and $D(T) \subset D\left(N_{1} N\right)$. Therefore

$$
\left(N x, N_{1}^{*} y\right)=\left(N_{1} N x, y\right)=\left(N_{1} N_{2} N_{1} x, y\right)=\left(N N_{1} x, y\right)=\left(N_{1} x, N^{*} y\right)
$$

for all $x, y \in D(T)$. It follows from Theorem 1 that $N$ permutes with $N_{1}$. Similarly, $N$ permutes with $N_{2}$. Let $\{E(\sigma)\}$ be the canonical spectral measure of $N$ and $E_{n}$ $=E\left(\sigma_{n}\right)$, where $\sigma_{n}=\{\lambda|n-1 \leqq| \lambda \mid<n\}$. Then $E_{n}$ permutes with $N_{1}$ and $N_{2}$ and $\left(N_{1} E_{n}\right)\left(N_{2} E_{n}\right)=N_{1} N_{2} E_{n}=N_{2} N_{1} E_{n}=\left(N_{2} E_{n}\right)\left(N_{1} E_{n}\right)$ for all $n$. Hence, by Proposition 2, $N_{1}$ and $N_{2}$ permute.

In [1] the author proved jointly with A. Devinatz and J. von Neumann that if $T$, $T_{1}$ and $T_{2}$ are selfadjoint operators such that $T \subset T_{1} T_{2}$, then $T_{1}$ and $T_{2}$ permute and $T=T_{1} T_{2}$.

This result will be proved below as a consequence of a more general formulation.

THEOREM 3. Let $T$ be a selfadjoint operator and $T_{1}, T_{2}$ closed symmetric operators such that $T_{0} \subset T_{1} T_{2}$, where $T_{0}$ is the restriction of $T$ to $D_{0}(T)$. Then $T_{2}$ is selfadjoint and its spectral measure permutes with $T$ and $T_{1}$.

Proof. $D_{0}(T) \subset D\left(T_{2}\right) \subset D\left(T_{2}^{*}\right)$,

$$
\left(T x, T_{2}^{*} y\right)=\left(T_{1} T_{2} x, T_{2} y\right)=\left(T_{2} x, T_{1} T_{2} y\right)=\left(T_{2} x, T y\right)
$$

for all $x, y \in D_{0}(T)$. Hence, by Corollary $1, T_{2}$ is selfadjoint and permutes with $T$.

Let $\{E(\sigma)\}$ be the canonical spectral measure of $T, \sigma_{n}=\{\lambda|| \lambda \mid \leqq n\}$ and $E_{n}=E\left(\sigma_{n}\right)$. Then $T E_{n}=T_{0} E_{n} \subset T_{1} T_{2} E_{n}$. But $T E_{n}$ has domain $H$. Hence $T E_{n}=T_{1} T_{2} E_{n}$. Now, $T E_{n}$ and $T_{2} E_{n}$ are bounded selfadjoint operators because $E_{n}$ permutes with $T$ and $T_{2}$ and the operators $T E_{n}$ and $T_{2} E_{n}$ have domain $H$. Hence,

$$
\left(T_{2} E_{n}\right) T_{1} \subset\left(T_{2} E_{n}\right) T_{1}^{*} \subset\left(T_{1}\left(T_{2} E_{n}\right)\right)^{*}=T_{1}\left(T_{2} E_{n}\right) .
$$

That is, $\left(T_{2} E_{n}\right) T_{1} \subset T_{1}\left(T_{2} E_{n}\right)$. From this follows that $F_{n}(\Delta) T_{1} \subset T_{1} F_{n}(\Delta)$ for all Borel sets $\Delta$, where $\left\{F_{n}(\Delta)\right\}$ is the canonical spectral measure of $T_{2} E_{n}$. Now, if $\left\{E_{2}(\sigma)\right\}$ is the canonical spectral measure of $T_{2}$, then $F_{n}(\Delta) \rightarrow E_{2}(\Delta)$ strongly as $n \rightarrow \infty$ for every Borel set $\Delta$ (for $F_{n}(\Delta)=E_{2}(\Delta) E_{n}$ if $0 \notin \Delta$ and $F_{n}(\Delta)=E_{2}(\Delta) E_{n}+\left(I-E_{n}\right.$ ) if $0 \in \Delta)$.

Let $\Delta$ be a Borel set in $R$ and $x \in D\left(T_{1}\right)$. Then $F_{n}(\Delta) x \rightarrow E_{2}(\Delta) x$ and $F_{n}(\Delta) T_{1} x$ $=T_{1} F_{n}(\Delta) x \rightarrow E_{2}(\Delta) T_{1} x$ as $n \rightarrow \infty$. Hence, since $T_{1}$ is closed, $E_{2}(\Delta) x \in D\left(T_{1}\right)$ and $T_{1} E_{2}(\Delta) x=E_{2}(\Delta) T_{1} x$. That is, $E_{2}(\Delta) T_{1} \subset T_{1} E_{2}(\Delta)$ for all Borel sets $\Delta$.

COROLLARY 2. If in the preceding theorem $T_{1}$ is selfadjoint, then $T$ is the closure of $T_{1} T_{2}$. This is the case if $T_{2}$ has an inverse.

Proof. If $T_{1}$ is selfadjoint, then the closure $T_{3}$ of $T_{1} T_{2}$ is selfadjoint because $T_{1}$ and $T_{2}$ permute (cf. [4, p. 45]). From $T_{0} \subset T_{3}$ follows that $T \subset T_{3}$. But a selfadjoint operator has no proper symmetric extension. Hence $T=T_{3}$. 
Suppose $T_{2}^{-1}$ exists. Let $x \in D\left(T T_{2}^{-1}\right)$ and $E_{n}$ as in Theorem 3. Then $E_{n} x \rightarrow x$ as $n \rightarrow \infty$. Now,

$$
E_{n} T T_{2}^{-1} x=T E_{n} T_{2}^{-1} x=T_{0} E_{n} T_{2}^{-1} x=T_{1} T_{2} E_{n} T_{2}^{-1} x
$$

and $E_{n} x=E_{n} T_{2} T_{2}^{-1} x=T_{2} E_{n} T_{2}^{-1} x$.

Therefore,

$$
E_{n} T T_{2}^{-1} x=T_{1} E_{n} x \rightarrow T T_{2}^{-1} x \text { as } n \rightarrow \infty .
$$

Hence, since $T_{1}$ is closed, $x \in D\left(T_{1}\right)$ and $T_{1} x=T T_{2}^{-1} x$; i.e., $T T_{2}^{-1} \subset T_{1}$. Therefore $A \subset T_{1}$, where $A$ is the closure of $T T_{2}^{-1}$. But $A$ is selfadjoint because $T$ and $T_{2}^{-1}$ are selfadjoint and permute. Hence, as above $A=T_{1}$.

COROLlaRY 3. If $T_{1}$ is selfadjoint and $T_{2}, T$ closed symmetric operators such that $T_{1} T_{2} \subset T$ and if $T_{2}$ has a bounded inverse (and therefore is selfadjoint), then $T$ is selfadjoint and $T_{2}$ permutes with $T_{1}$ and $T$. Moreover $T=T_{2} T_{1}$.

(This result was obtained in [1] under the additional assumption that $T$ is selfadjoint.)

Proof. From the hypothesis we immediately get $T_{1} \subset T T_{2}^{-1}$. Since $T_{2}^{-1}$ has an inverse it follows from Corollary 2 and Theorem 3 that $T$ is selfadjoint, $T_{2}^{-1}$ permutes with $T$ and $T_{1}$, and $T_{1}=T T_{2}^{-1}$ (since $T T_{2}^{-1}$ is closed). Hence $T_{2}^{-1} T$ $\subset T T_{2}^{-1}=T_{1}$ and consequently $T \subset T_{2} T_{1}$. But $T$ is the closure of $T_{2} T_{1}$ by Corollary 2 . Hence $T=T_{2} T_{1}$.

The following theorem was proved by E. Nelson in [5] by entirely different and more complicated methods.

THEOREM 4. Let $A$ and $B$ be symmetric operators in a Hilbert space $\mathfrak{S}$ and let $\mathfrak{M}$ be a dense linear manifold in $\mathfrak{S}$ such that $\mathfrak{M}$ is contained in the domain of $A^{2}, B^{2}, A B$ and $B A$, and such that $A B x=B A x$ for all $x$ in $\mathfrak{M}$. If the restriction of $A^{2}+B^{2}$ to $\mathfrak{M}$ is essentially selfadjoint then $A$ and $B$ are essentially selfadjoint and $\bar{A}$ and $\bar{B}$ commute.

Proof. Let $T$ be the closure of the restriction of $A^{2}+B^{2}$ to $\mathfrak{M}$. Then $T$ is selfadjoint by hypothesis. Now, $D(T) \subset D(\bar{A})$. More specifically, if $x_{n} \in \mathfrak{M}, x_{n} \rightarrow x$ and $\left(A^{2}+B^{2}\right) x_{n} \rightarrow T x$ as $n \rightarrow \infty$, then $A x_{n} \rightarrow \bar{A} x$ as $n \rightarrow \infty$. Indeed,

$$
\left(\left(A^{2}+B^{2}\right)\left(x_{n}-x_{m}\right), x_{n}-x_{m}\right)=\left\|A\left(x_{n}-x_{m}\right)\right\|^{2}+\left\|B\left(x_{n}-x_{m}\right)\right\|^{2} \rightarrow 0
$$

as $n, m \rightarrow \infty$. Hence $\left(A x_{n}\right)_{n \geqq 1}$ converges to, say, $y$ as $n \rightarrow \infty$. Hence, $x \in D(\bar{A})$ and $\bar{A} x=y$; i.e., $A x_{n} \rightarrow \bar{A} x$ as $n \rightarrow \infty$. By symmetry the same is true if $A$ is replaced by $B$.

Let $x$ and $y$ be elements of $D(T)$. Choose elements $x_{n}$ and $y_{n}$ in $\mathfrak{M}$ such that $x_{n} \rightarrow x,\left(A^{2}+B^{2}\right) x_{n} \rightarrow T x$ and $y_{n} \rightarrow y,\left(A^{2}+B^{2}\right) y_{n} \rightarrow T y$, as $n \rightarrow \infty$. Then

$$
\begin{aligned}
\left(T x, A^{*} y\right)=(T x, \bar{A} y) & =\lim _{n \rightarrow \infty}\left(\left(A^{2}+B^{2}\right) x_{n}, A y_{n}\right) \\
& =\lim _{n \rightarrow \infty}\left(A x_{n},\left(A^{2}+B^{2}\right) y_{n}\right)=(\bar{A} x, T y),
\end{aligned}
$$


by repeated use of the relation $A B z=B A z$ for all $z \in \mathfrak{M}$. Hence $\bar{A}$ is selfadjoint and permutes with $T$ by Corollary 1 . Similarly $\bar{B}$ is selfadjoint and permutes with $T$.

Let $\{E(\sigma)\}$ be the canonical spectral measure of $T$ and $E_{n}=E\left(\sigma_{n}\right)$, where $\sigma_{n}$ $=\{\lambda|n-1 \leqq| \lambda \mid<n\}$. Then $E_{n}$ permutes with $\bar{A}$ and $\bar{B}$ and $R\left(E_{n}\right) \subset D(T) \subset D(\bar{A})$. Thus $R\left(E_{n}\right) \subset D(\bar{A})$. Similarly $R\left(E_{n}\right) \subset D(\bar{B})$. It follows that $\bar{A} E_{n}$ and $\bar{B} E_{n}$ are bounded selfadjoint operators. We assert that $A_{n}=\bar{A} E_{n}$ and $B_{n}=\bar{B} E_{n}$ permute. Indeed, let $x$ be any element in $\mathfrak{S}$. Then $E_{n} x \in D(T)$. Now choose a sequence $\left(y_{k}\right)$ in $\mathfrak{M}$ such that $y_{k} \rightarrow E_{n} x$ and $\left(A^{2}+B^{2}\right) y_{k} \rightarrow T E_{n} x$ as $k \rightarrow \infty$. Then, as was shown above, $A y_{k} \rightarrow \bar{A} E_{n} x$ and $B y_{k} \rightarrow \bar{B} E_{n} x$ as $k \rightarrow \infty$. Hence

$$
\begin{aligned}
A_{n} B_{n} x & =\lim _{k \rightarrow \infty}\left(\bar{A} E_{n}\right) B y_{k}=\lim _{k \rightarrow \infty} E_{n} A B y_{k} \\
& =\lim _{k \rightarrow \infty} E_{n} B A y_{k}=\lim _{k \rightarrow \infty}\left(\bar{B} E_{n}\right) A y_{k}=B_{n} A_{n} x .
\end{aligned}
$$

That $\bar{A}$ and $\bar{B}$ permute now follows from Proposition 2 .

In [5] E. Nelson proved the existence of two symmetric operators $A$ and $B$ in a Hilbert space $\mathfrak{S}_{\mathfrak{C}}$ having a common invariant domain $\mathfrak{D}$ such that for all real $a$ and $b$, $a A+b B$ is essentially selfadjoint and such that for all $x$ in $\mathfrak{D}, A B x=B A x$, but such that $\bar{A}$ and $\bar{B}$ do not commute.

We shall show in the next theorem that there are no symmetric operators with the described properties if we require in addition that the domain of the closure of the operator $A+B$ is contained in the domain of $\bar{A}$ or $\bar{B}$.

More precisely we have the following theorem.

THEOREM 5. Let $A$ and $B$ be symmetric operators in a Hilbert space $\mathfrak{S}$ and let $\mathfrak{M}$ be a dense linear manifold in $\mathfrak{H}$ such that $\mathfrak{M}$ is contained in the domain of $A$ and $B$, and such that $(A x, B y)=(B x, A y)$ for all $x$ and $y$ in $\mathfrak{M}$. Let $T$ be the closure of the restriction of $A+B$ to $\mathfrak{M}$. If T is selfadjoint and $D(T) \subset D(\bar{A})$ then $A$ and $B$ are essentially selfadjoint and $\bar{A}$ and $\bar{B}$ commute. This condition is satisfied, for example, if there exists a number $c$ such that $(A x, B x) \geqq c\|x\|^{2}$ for all $x$ in $\mathfrak{M}$.

Proof. The proof is analogous to the proof of Theorem 4. Let $x \in D(T)$ and $\left(x_{n}\right)$ be a sequence of elements in $\mathfrak{M}$ such that $x_{n} \rightarrow x$ and $(A+B) x_{n} \rightarrow T x$ as $n \rightarrow \infty$. We shall show that $A x_{n} \rightarrow \bar{A} x$ as $n \rightarrow \infty$.

To see this, consider the mapping $(x, T x) \rightarrow \bar{A} x$ of the graph $\mathscr{G}(T)$ of $T$ into $\mathfrak{H}$. This linear transformation is closed since $\bar{A}$ is a closed operator. $\mathscr{G}(T)$ is a closed subspace of $\mathfrak{S} \times \mathfrak{S E}$ and therefore a Banach space. It follows from the closed graph theorem that the mapping $(x, T x) \rightarrow \bar{A} x$ is continuous. Hence there exists a constant $a$ such that

$$
\|\bar{A} x\| \leqq a\left(\|x\|^{2}+\|T x\|^{2}\right)^{1 / 2} \text { for all } x \in D(T) .
$$

From the last inequality follows that $\left\|\bar{A}\left(x_{n}-x_{m}\right)\right\| \rightarrow 0$ as $n, m \rightarrow \infty$. That is, $x_{n} \rightarrow x$ and $A x_{n}=\bar{A} x_{n} \rightarrow y$ as $n \rightarrow \infty$, where $y$ is some element in $\mathfrak{S}$. Hence $x \in D(\bar{A})$ and $\bar{A} x=y$, since $\bar{A}$ is closed. 
If $x$ and $y$ are elements in $D(T)$, choose sequences $\left(x_{n}\right)$ and $\left(y_{n}\right)$ in $\mathfrak{M}$ such that $x_{n} \rightarrow x,(A+B) x_{n} \rightarrow T x$ and $y_{n} \rightarrow y,(A+B) y_{n} \rightarrow T y$, as $n \rightarrow \infty$. Then

$$
\begin{aligned}
\left(T x, A^{*} y\right) & =(T x, \bar{A} y) \\
& =\lim _{n \rightarrow \infty}\left((A+B) x_{n}, A y_{n}\right)=\lim _{n \rightarrow \infty}\left(A x_{n},(A+B) y_{n}\right) \\
& =(\bar{A} x, T y) .
\end{aligned}
$$

Hence, by Corollary $1, \bar{A}$ is selfadjoint and permutes with $T$. Similarly $\bar{B}$ is selfadjoint and permutes with $T$, for $D(T) \subset D(\bar{B})$ as shown further down.

To show that $\bar{A}$ and $\bar{B}$ commute consider the canonical spectral measure $\{E(\sigma)\}$ of $T$. Let $E_{n}=E\left(\sigma_{n}\right)$, where $\sigma_{n}=\{\lambda|n-1 \leqq| \lambda \mid<n\}$. Then $E_{n}$ permutes with $\bar{A}$ and $\bar{B}$ and $R\left(E_{n}\right) \subset D(T) \subset D(\bar{A})$. If $x \in D(T)$ choose a sequence $\left(x_{n}\right)$ in $\mathfrak{M}$ such that $x_{n} \rightarrow x$ and $(A+B) x_{n} \rightarrow T x$ as $n \rightarrow \infty$. Then $A x_{n} \rightarrow \bar{A} x$ as we have seen. Hence $B x_{n}=(A+B) x_{n}-A x_{n} \rightarrow T x-\bar{A} x$ as $n \rightarrow \infty$. Therefore, since $\bar{B}$ is closed, $x \in D(\bar{B})$ and $\bar{B} x=T x-\bar{A} x$; i.e., $T x=\bar{A} x+\bar{B} x$. Thus $T \subset \bar{A}+\bar{B}$. From this follows, since $\bar{A}+\bar{B}$ is symmetric and $T$ is selfadjoint that $T=\bar{A}+\bar{B}$. It follows that $R\left(E_{n}\right) \subset D(\bar{B})$ and $T_{n}=A_{n}+B_{n}$, where $T_{n}=T E_{n}, A_{n}=\bar{A} E_{n}$ and $B_{n}=\bar{B} E_{n}$ are bounded selfadjoint operators. $A_{n}$ permutes with $T_{n}$ and hence with $B_{n}=T_{n}-A_{n}$. It follows now from Proposition 2 that $\bar{A}$ and $\bar{B}$ permute. (The fact that $\bar{A}$ and $\bar{B}$ commute can also be proved as in the proof of Theorem 4.)

Finally, suppose there is a number $c$ such that $(A x, B x) \geqq c\|x\|^{2}$ for all $x \in \mathfrak{M}$. Let $x \in D(T)$ and choose a sequence $\left(x_{n}\right)$ in $\mathfrak{M}$ such that $x_{n} \rightarrow x$ and $(A+B) x_{n} \rightarrow T x$ as $n \rightarrow \infty$. Then

$$
\begin{aligned}
\left\|(A+B)\left(x_{n}-x_{m}\right)\right\|^{2} & =\left\|A\left(x_{n}-x_{m}\right)\right\|^{2}+\left\|B\left(x_{n}-x_{m}\right)\right\|^{2}+2\left(A\left(x_{n}-x_{m}\right), B\left(x_{n}-x_{m}\right)\right) \\
& \geqq\left\|A\left(x_{n}-x_{m}\right)\right\|^{2}+2 c\left\|x_{n}-x_{m}\right\|^{2} .
\end{aligned}
$$

Therefore $\left\|A\left(x_{n}-x_{m}\right)\right\| \rightarrow 0$ as $n, m \rightarrow \infty$. It follows, since $\bar{A}$ is closed, that $x \in D(\bar{A})$.

\section{REFERENCES}

1. A. Devinatz, A. E. Nussbaum and J. von Neumann, On the permutability of selfadjoint operators, Ann. of Math. (2) 62 (1955), 199-203.

2. A. Devinatz and A. E. Nussbaum, On the permutability of normal operators, Ann. of Math. (2) 65 (1957), 144-152.

3. B. Fuglede, A commutativity theorem for normal operators, Proc. Nat. Acad. Sci. 36 (1950), 35-40.

4. B. v. Sz. Nagy, Spektraldarstellung linearer Transformationen des Hilbertschen Raumes, Springer, Berlin, 1942.

5. E. Nelson, Analytic vectors, Ann. of Math. (2) 70 (1959), 572-615.

6. B. Fuglede, Problem 3, Math. Scand. 1 (1953), 172 and 2 (1954), 346-347.

WASHINGTON UNIVERSITY,

St. Louis, MissourI

STANFORd UNIVERSITY,

Stanford, California 